\title{
CHOROIDAL MELANOMA PRESENTING AS VITREOUS HAEMORRHAGE
}

\author{
I. A. CUNLIFFE and I. G. RENNIE \\ Sheffield
}

\begin{abstract}
SUMMARY
Vitreous haemorrhage is an unusual presenting feature of malignant melanoma of the choroid. We describe three cases in which this occurred. The three tumours shared a number of common features: all were located in the peripheral choroid, were of a similar size and had a small herniation of tumour tissue through Bruch's membrane. We believe that vascular congestion caused by constriction of the tumour vasculature at the site of herniation is the most probable cause for the haemorrhage in these cases. We review the relevant literature and discuss some of the commoner differential diagnoses.
\end{abstract}

Vitreous haemorrhage is an unusual presentation of malignant melanoma of the choroid. In one ultrasound study of acute vitreous haemorrhage, ${ }^{1}$ malignant melanoma was responsible for $2 \%$ of cases. In a pathological study $2 \%$ of eyes enucleated for melanoma had vitreous haemorrhage as their presenting feature. In this paper, three cases of malignant melanoma of the choroid that presented with vitreous haemorrhage are described in detail. The differential diagnosis is discussed and the possible mechanisms of aetiology are reviewed.

\section{CASE REPORTS}

\section{Case 1}

A 62-year old woman presented with sudden reduction of the vision in her right eye. On examination she had normal anterior segments and intraocular pressures, but ophthalmoscopy revealed a right vitreous haemmorhage and a suspicious lesion in the lower nasal periphery. The haemorrhage gradually cleared with a corresponding improvement in vision. She was referred to our unit for further assessment. On examination at that stage she had acuities of 6/6 right and 6/5 left. Anterior segments and intraocular pressures were normal. Ophthalmoscopy revealed the presence of residual vitreous haemorrhage in the right eye

From: Department of Ophthalmology and Orthoptics, University of Sheffield, Sheffield, UK.

Correspondence to: I. A. Cunliffe, Department of Ophthalmology and Orthoptics, O Floor, Royal Hallamshire Hospital, Glossop Road, Sheffield S10 2JF, UK. with an elevated lesion in the infero-nasal periphery. B-scan ultrasound examination confirmed the presence of a solid lesion measuring $11.1 \times 9.3 \mathrm{~mm}$ in diameter with a height of $4.9 \mathrm{~mm}$, with choroidal excavation. There was a small herniation through Bruch's membrane at the apex of the tumour (Fig. 1), and vitreous haemorrhage and retinal detachment were also present. A-scan examination showed foci of high internal reflectivity. After further clearing of the vitreous haemorrhage the lesion was clinically confirmed as an amelanotic malignant melanoma. The tumour was treated with ruthenium plaque brachytherapy.

\section{Case 2}

An 80-year-old man was referred to our unit with a 6 month history of sudden reduction in visual acuity in his right eye which more recently had started to improve. At his initial presentation he had normal anterior segments and intraocular pressures and ophthalmoscopic examination revealed the cause of the blurred vision to be a vitreous haemorrhage that appeared to be arising from an elevated lesion in the upper temporal fundus. On examination in this unit, he had acuities of 6/9 right and left. Anterior segments and intraocular pressures were normal. Ophthalmoscopy revealed an elevated lesion arising from supero-temporal choroid with herniation through Bruch's membrane, and some residual vitreous haemorrhage.

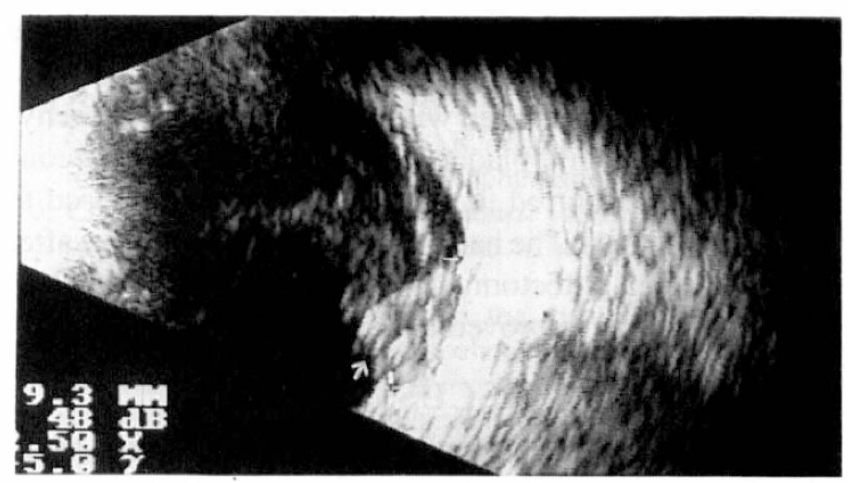

Fig. 1. Ultrasound scan showing a raised solid lesion with herniation through Bruch's membrane (arrowed). Retinal detachment and vitreous haemorrhage are also shown. 


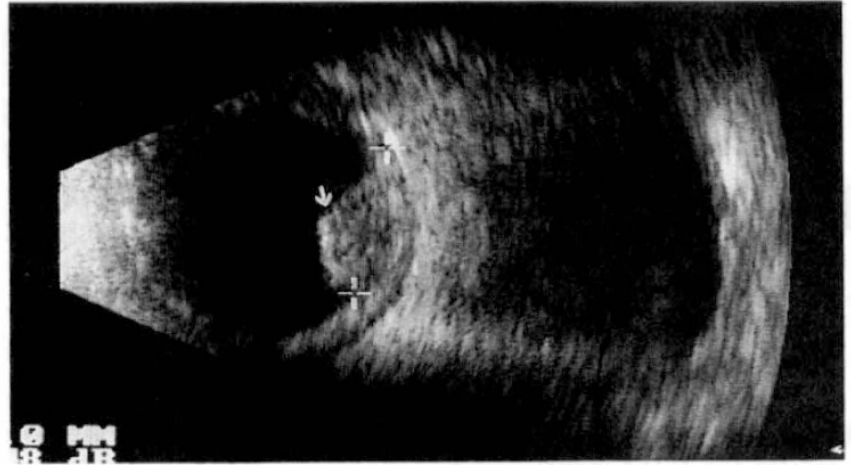

Fig. 2. Ultrasound scan showing a raised solid lesion with herniation through Bruch's membrane at its apex (arrowed). This scan was done after the insertion of the ruthenium plaque (which can be seen) and this accounts for the orbital shadowing.

B-scan ultrasound showed a dome-shaped lesion measuring $11.5 \times 12 \mathrm{~mm}$ in diameter and $5.8 \mathrm{~mm}$ in height. There was a small herniation through Bruch's membrane at the apex of the tumour (Fig. 2). The lesion was consistent with a malignant melanoma, and was treated with ruthenium plaque brachytherapy.

\section{Case 3}

A 79-year-old man presented with a 1 month history of reduced vision in the left eye. On examination he had a raised intraocular pressure and cupped optic disc in the right eye but a normal intraocular pressure and anterior segment in the left eye. Ophthalmoscopy revealed the presence of a vitreous haemorrhage in the left eye with a suspicious raised lesion in the infero-temporal quadrant. $\mathrm{He}$ was referred to our unit for further assessment. On examination at that stage he had acuities of 6/60 right and $6 / 36$ left; anterior segments showed bilateral corneal guttata but were otherwise normal. Intraocular pressures were controlled on treatment. Ophthalmoscopy confirmed the vitreous haemorrhage and showed an elevated lesion in the infero-temporal fundus that had broken through Bruch's membrane. B-scan ultrasound showed the lesion to be $10.2 \times 8.3 \mathrm{~mm}$ in diameter with a height of $5.6 \mathrm{~mm}$. There was a narrowing at the midpoint of the tumour indicating herniation through Bruch's membrane and vitreous haemorrhage was also present (Fig. 3). The lesion had low internal reflectivity on A-scan examination.

The tumour was treated with ruthenium plaque brachytherapy. Following plaque therapy a further vitreous haemorrhage occurred in the eye with vision reduced to Hand Movements. The haemorrhage failed to resolve after 8 months and a vitrectomy was performed. Following this the visual acuity improved to $6 / 12$.

\section{DISCUSSION}

Dense vitreous haemorrhage with sudden decrease in visual acuity is an unusual presenting feature of malignant melanoma of the choroid. Hasenfratz $z^{1}$ in an ultrasound study of 216 eyes presenting with acute vitreous haemorrhage found 5 eyes (2\%) to have a malignant melanoma of the choroid. The commonest causes of acute vit- reous haemorrhage in this study were diabetic retinopathy $(42 \%)$, posterior vitreous detachment $(27 \%)$ and disciform macular degeneration (18\%). In another study ${ }^{2} 9$ of 450 eyes $(2 \%)$ enucleated for intraocular melanoma had vitreous haemorrhage as the presenting feature. Seven of these eyes had a choroidal melanoma, 1 contained a ciliary body melanoma and 1 was described as 'diffuse'. Conversely, Shields et al. ${ }^{3}$ reported that vitreous haemorrhage was misdiagnosed as a melanoma in $2.8 \%$ of cases.

The commoner haemorrhagic lesions that may be mistaken for a choroidal melanoma include macular and extramacular disciforms, haemorrhagic choroidal detachments and arteriolar macroaneurysms. Macular and extramacular haemorrhagic disciforms were the second and third commonest lesions simulating melannomas in the study reported by Shields et al. ${ }^{3}$ Haemorrhagic detachments of the retina or retinal pigment epithelium at the macular area can simulate melanomas. Haemorrhage from these lesions can be subretinal, retinal or vitreal depending on the extent of the bleeding. Secondary haemorrhagic detachments at the edge of old disciform scars may also simulate melanomas. These lesions are well reported and have been reviewed by Gass. ${ }^{4}$

Extramacular disciform lesions are less common, but are one of the main differential diagnoses for a peripheral malignant melanoma. In a series of extramacular disciform lesions simulating uveal tumours, Bardenstein et al. ${ }^{5}$ discuss several features that help in the differential diagnosis. Vitreous haemorrhage was much more common in the disciform lesions than in choroidal melanomas and the source of the haemorrhage was also different. In extramacular disciform lesions the haemorrhage usually originates from the base of the lesion. Extramacular disciforms tend to be flat with irregular edges and shrink in size when examined over time, whereas melanomas tend to be dome-shaped or have a collar-stud appearance. In their series ultrasound examination had a diagnostic accuracy of $83 \%$, with the characteristics of extramacular disciforms being distinct from those of uveal melanomas. None of the disciform lesions had acoustic shadowing or spontaneous pulsations, they had more heterogeneity, and the junction between tumour and sclera tended to be 'climbing' rather than vertical as seen in melanomas.

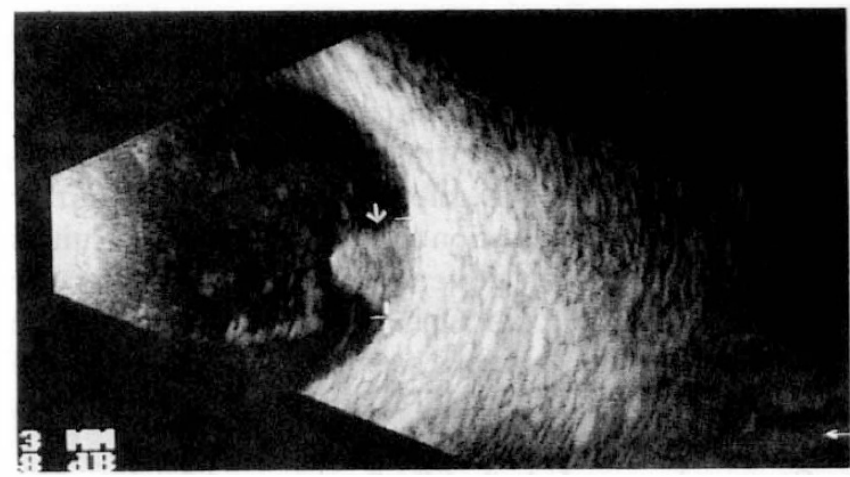

Fig. 3. Ultrasound scan showing a raised solid lesion with evidence of choroidal excavation. There is narrowing at the midpoint of the tumour (arrowed) indicating herniation through Bruch's membrane. Vitreous haemorrhage can also be seen. 
Fluorescein angiography was diagnostically useful in the cases in which the media were clear enough to allow photography.

Post-operative choroidal detachments may occasionally simulate melanomas but can usually be distinguished with transillumination, ultrasound and periodic observation. Haemorrhagic choroidal detachments may be more difficult to distinguish as they can cast a shadow on transillumination and ultrasound can show internal reflectivity. ${ }^{6}$ Conversely, malignant melanomas can simulate choroidal detachment. ${ }^{7}$

Rupture of arterial aneurysms can lead to large retinal, subretinal or vitreous haemorrhage and the appearance may simulate melanomas clinically. Perry et al. ${ }^{8}$ have reported two such cases. Ultrasound and fluorescein angiography should help to distinguish between these lesions.

In a prospective study of 51 histologically confirmed choroidal melanomas Char et al. ${ }^{9}$ examined the accuracy of various diagnostic modalities used in the investigation of suspected melanoma. Ophthalmoscopic evaluation was found to be the most accurate for tumour diagnosis, with all tumours being correctly diagnosed in eyes with clear media. Ultrasound examination was suggestive or diagnostic of melanoma in $89 \%$ of cases and fluorescein angiography in $70 \%$ of cases. In eyes in which the media are not clear, as in the three cases reported in this study, clinical assessment is more difficult and fluorescein angiography is not possible. In these cases ultrasound is the most useful aid to diagnosis.

When a tumour breaks Bruch's membrane, a small retinal or tumour vessel may tear, resulting in a small localised vitreous haemorrhage. However, large dense vitreous haemorrhage due to tumour invading through Bruch's membrane is uncommon. ${ }^{10}$ There may be several mechanisms by which malignant melanomas may produce vitreous haemorrhage. In a case described by Gass" he postulates that a small hole in Bruch's membrane may lead to a constricting effect on the tumour vessels with a subsequent increase in venous pressure within the tumour. The dilated vessels on the tumour surface may then bleed into the vitreous. Jensen ${ }^{12}$ described a tumour that had grown through Bruch's membrane and had a spherical vascular head with a cavernous structure that may have accounted for the large vitreous haemorrhage. Kielar ${ }^{13}$ has reported a case presenting with dense vitreous haemorrhage in which the source of the haemorrhage was thought to be large vascular cavities within the tumour. There was disruption of Bruch's membrane in two areas of this tumour, but tumour extension through Bruch's membrane had occurred at only one of these areas. Yee et al. ${ }^{14}$ described a case in which invasion and occlusion of a choroidal artery by tumour cells had led to coagulative necrosis of the tumour with a subsequent haemorrhage that had broken through Bruch's membrane but remained subretinal. This may be another mechanism by which melanomas can lead to a large vitreous haemorrhage.

In this study the three tumours shared a number of com- mon features: all were located in the peripheral choroid, were of a similar size and had a small herniation of tumour tissue through Bruch's membrane. The lack of any haemorrhage at the tumour base or on the surface beneath Bruch's membrane supports the belief that the vitreous haemorrhage had arisen from the portion of the tumour which had invaded Bruch's membrane. We believe that vascular congestion caused by constriction of the tumour vasculature at the site of herniation is the most probable cause for the haemorrhage in these cases.

In conclusion, vitreous haemorrhage is an unusual presenting feature of malignant melanoma of the choroid. It probably occurs mainly in tumours that have broken through Bruch's membrane in which the vessels may be constricted by the membrane resulting in vascular congestion. Other lesions producing vitreous haemorrhage may be mistaken for malignant melanoma; however, with current diagnostic techniques the rate of misdiagnosis can be minimised.

Key words: Choroidal melanoma, Vitreous haemorrhage.

\section{REFERENCES}

1. Hasenfratz G. Acute vitreous haemorrhage: possibilities for differential diagnostic, echographic assessment. Fortschr Ophthalmol 1990;87:641-5.

2. Fraser DJ, Font RL. Ocular inflammation and haemorrhage as initial manifestations of uveal malignant melanoma. Arch Ophthalmol 1979;97:1311-4.

3. Shields JA, Augsburger JJ, Brown GC, et al. The differential diagnosis of posterior uveal melanoma. Ophthalmology 1980;87:543-8.

4. Gass JDM. Pathogenesis of disciform detachment of the neuroepithelium. III. Senile disciform macular degeneration. Am J Ophthalmol 1967;63:617.

5. Bardenstein DS, Char DH, Irvine AR, Stone RD. Extramacular disciform lesions simulating uveal tumors. Ophthalmology 1992;99:944-51.

6. Shields JA, Shields CL. Differential diagnosis of posterior uveal melanoma. In: Shields JA, Shields CL, editors. Intraocular tumours: a text and atlas. Philadelphia: WB Saunders, 1992:137-53.

7. Shields JA, Leonard BC, Sarin LK. Multilobed uveal melanoma masquerading as a postoperative choroidal detachment. Br J Ophthalmol 1976;60:386-9.

8. Perry HD, Zimmermann LE, Benson WE. Hemorrhage from isolated aneurysm of a retinal artery: report of two cases simulating malignant melanoma. Arch Ophthalmol 1977;95:281-3

9. Char DH, Stone RD, Irvine AR, et al. Diagnostic modalities in choroidal melanoma. Am J Ophthalmol 1980;89:223-30.

10. Char DH. Posterior uveal tumours. In: Char DH. Clinical ocular oncology. New York: Churchill Livingstone, 1989:91-149.

11. Gass JDM. Hemorrhage into the vitreous, a presenting manifestation of malignant melanoma of the choroid. Arch Ophthalmol 1963;69:778-9.

12. Jensen OA. Malignant melanoma of the choroid of a peculiar cavernous type. Arch Ophthalmol 1964;72:337-40.

13. Kielar RA. Choroidal melanoma presenting as vitreous hemorrhage. Ann Ophthalmol 1982;14:461-4.

14. Yee RD, Foos RY, Straatsma BR. Coagulative necrosis in a malignant melanoma of the choroid at the macula with extensive subretinal haemorrhage. Invest Ophthalmol $1973 ; 12: 525-31$ 